\title{
Wireless deep-subwavelength metamaterial enabling sub-mm resolution magnetic resonance imaging
}

\author{
Sayim Gokyar ${ }^{\mathrm{a}, \mathrm{b}, *}$, Akbar Alipour $^{\mathrm{a}, \mathrm{b}}$, Emre Unal $^{\mathrm{a}, \mathrm{b}}$, Ergin Atalar $^{\mathrm{a}}$, Hilmi Volkan Demir ${ }^{\mathrm{a}, \mathrm{b}, \mathrm{c}}$ \\ a Department of Electrical and Electronics Engineering, Department of Physics, National Magnetic Resonance Research Center (UMRAM), Bilkent University, \\ Ankara, TR-06800, Turkey \\ b UNAM-National Nanotechnology Research Center and Institute of Materials Science and Nanotechnology, Bilkent University, Ankara, TR-06800, Turkey \\ ${ }^{\mathrm{c}}$ Luminous! Center of Excellence for Semiconductor Lighting and Displays, School of Electrical and Electronic Engineering, School of Physical and \\ Mathematical Sciences, Physics and Applied Physics Division, Nanyang Technological University, Singapore
}

\section{A R T I C L E I N F O}

\section{Article history:}

Received 9 May 2017

Received in revised form 4 March 2018

Accepted 19 March 2018

Available online 20 March 2018

Keywords:

Magnetic resonance imaging

Metamaterials

Deep-subwavelength resonators

\begin{abstract}
A B S T R A C T
A wireless deep-subwavelength metamaterial architecture is proposed, modeled and demonstrated for a high-resolution magnetic resonance imaging (HR-MRI) application that is miniaturized to be resonant at approximately $\lambda_{0} / 1500$ dimensions. The proposed structure has the adjustable resonance frequency from $65 \mathrm{MHz}$ to $5.5 \mathrm{GHz}$ for the sub-cm footprint area $(8 \mathrm{~mm} \times 8 \mathrm{~mm}$ for this study) and provides a quality factor (Q-factor) of approximately 80 in free space for $123 \mathrm{MHz}$ of operation. This structure consists of a cross-via metallized partial-double-layer metamaterial, sandwiching a dielectric thin film; this structure strongly localizes the electric field in this film and has a highly capacitive metal overlay that allows for a wide range of frequency adjustment. Although the achieved resonance frequencies enable a large number of applications, as a proof-of-concept demonstration, we experimentally showed the operation of this wireless metastructure in HR-MRI to highlight its precise frequency adjustment and signal-to-noise-ratio (SNR) improvement capabilities. The proposed metamaterial was found to maintains high Q-factors despite loading with a body-mimicking lossy phantom. The experimental results indicated that the proposed metastructure can be used as an SNR-enhancing device offering 15-fold SNR enhancements that allows for imaging of objects as small as $200 \mu \mathrm{m}$ in diameter in its vicinity, at an unprecedented level of resolution at the given DC field using standard head coils. As a result of its deep-subwavelength miniaturization accompanied by reasonable Q-factor with outstanding resonance frequency adjustment capability, this class of metastructure is proved to be an excellent candidate for in vivo medical applications.
\end{abstract}

(c) 2018 Elsevier B.V. All rights reserved.

\section{Introduction}

Following the seminal work of Veselago [1], metamaterials have been introduced in different applications including material characterization [2], sensing [3-8], compacting devices [9] and imaging of subwavelength features [10], spanning from optical frequencies [11] to the radiofrequency (RF) region [12]. The operating bandwidth of those metamaterial devices has been typically narrow because of the high quality factor ( $Q$-factor) elements used in the unit cell of their structures. Because magnetic resonance imaging (MRI) is basically a narrow bandwidth imaging technique, metamaterials could be quite attractive for use in MRI applications. It

\footnotetext{
* Corresponding author at: Department of Electrical and Electronics Engineering, Department of Physics, National Magnetic Resonance Research Center (UMRAM), Bilkent University, Ankara, TR-06800, Turkey.

E-mail address: sayim@ee.bilkent.edu.tr (S. Gokyar).
}

was previously shown that metamaterial structures can be used for RF flux guiding [13] and signal-to-noise-ratio (SNR) improvement purposes [14]. These metamaterials, in addition to conventional imaging hardware of the scanner, properly manipulates the EM fields in their vicinity to increase imaging signal emitted from the imaged objects (e.g., tissues). However, these wireless designs are larger in size to be used for medical applications; hence, the design of wireless sub-cm metamaterials to address in vivo MRI of sub-mm features has not been demonstrated to date.

High-resolution MRI (HR-MRI) suffers the fundamental problem of reduced SNR because of the decreased volume of the imaged voxels. To increase the SNR of an MR image, several methods can be applied. These include using a higher DC magnetic field [15], using high density coil arrays with parallel imaging techniques $[16,17]$, increasing the number of image acquisition and using highsensitivity wired coils [18-27]. Practically, the DC field strength is predetermined and assumed to be constant after the installment of the main magnet. However, using high density coil arrays with par- 
allel imaging techniques is one of the major breakthroughs; their performances can be increased by using wireless coils for in vivo applications. Increasing the number of acquired images increases the imaging duration and total RF power exposure of patients, which is not suitable for clinical imaging practices. Hence, for a predetermined DC field and a receiver coil configuration, increasing SNR without increasing the total RF power exposure and imaging duration becomes possible with localized coil solutions. Unfortunately, these coils [18-28] require complicated electronics (e.g., matching capacitors, solder, diodes and/or cryo-cooling etc.) and they are connected to the body of the scanner via RF cables. Hence, their application to in vivo operations is inherently challenging because of RF heating risks [29]. Using wireless resonators for in vivo MRI applications would increase the detection performance of an MRI system [13, 14, 30-32]. There has been significant effort in the literature to decrease the resonance frequency $\left(f_{0}\right)$ of metamaterials [33] such as, increasing the size of the elements given in the slab [34] and/or using lumped capacitors [12] to capacitively load the unit cells. Although most of these methods are acceptable for certain applications, such as strain sensing [35], they are not suitable for in vivo applications because of their size [34] and lumped capacitors used to tune their resonance frequencies [12]. However, none of the previous wireless designs, or metamaterials, achieved the electrical size of smaller than $\lambda / 300$ and a $Q$-factor of more than 50 simultaneously without using cryo-cooling or a lumped element thus far.

In this work, to address the aforementioned problems of HR-MRI, we demonstrate a wireless deep-subwavelength metastructure enabling a Q-factor of approximately 80 in free space, in a highly compact footprint area. Here the proposed wireless metamaterial structure, while being electrically very small, is also shown to be an excellent candidate for in vivo MRI applications including HR-MRI at sub-mm resolution.

\section{Methods}

The proposed structure is a cross-via metallized double-layer metamaterial, in which the consecutive metal layers are strongly coupled (i.e. both inductively and capacitively) to each other through an overlay region to decrease the resonance frequency to a pre-defined frequency. Fig. 1.a shows a schematic of the proposed structure. Conductive cross-via metallization results in a thinfilm loaded semi-turn over-laid double-layer resonator. Unlike the classical split ring resonators (SRR) and multi-layer SRRs, this metamaterial structure exhibits higher inductive coupling via this cross-via metallization between consecutive layers.

\subsection{Equivalent circuit modeling and full-wave numerical analysis}

Equivalent circuit models of SRRs [36] and spirals [37] have been previously reported in the literature, where the single layer resonators are modeled as a series RLC circuit with proper mutual coupling terms. Resonance frequency of a resonator is given by Eq. (1)

$f_{0}=\frac{1}{2 \pi \sqrt{L_{e f f} C_{e f f}}}$

where $L_{e f f}$ is the effective inductance and $C_{\text {eff }}$ is the effective capacitance of the overall structure. Q-factor of a series resonator is given by Eq. (2)

$Q=\frac{2 \pi f_{0} L_{e f f}}{R_{e f f}}=\frac{1}{R_{e f f}} \sqrt{\frac{L_{e f f}}{C_{e f f}}}=\frac{f_{0}}{f_{3 d B}}$

where $R_{e f f}$ is the effective resistance and $\mathrm{f}_{3 \mathrm{~dB}}$ is the full-width-halfmaximum (FWHM) bandwidth of the resonator. Unlike previously
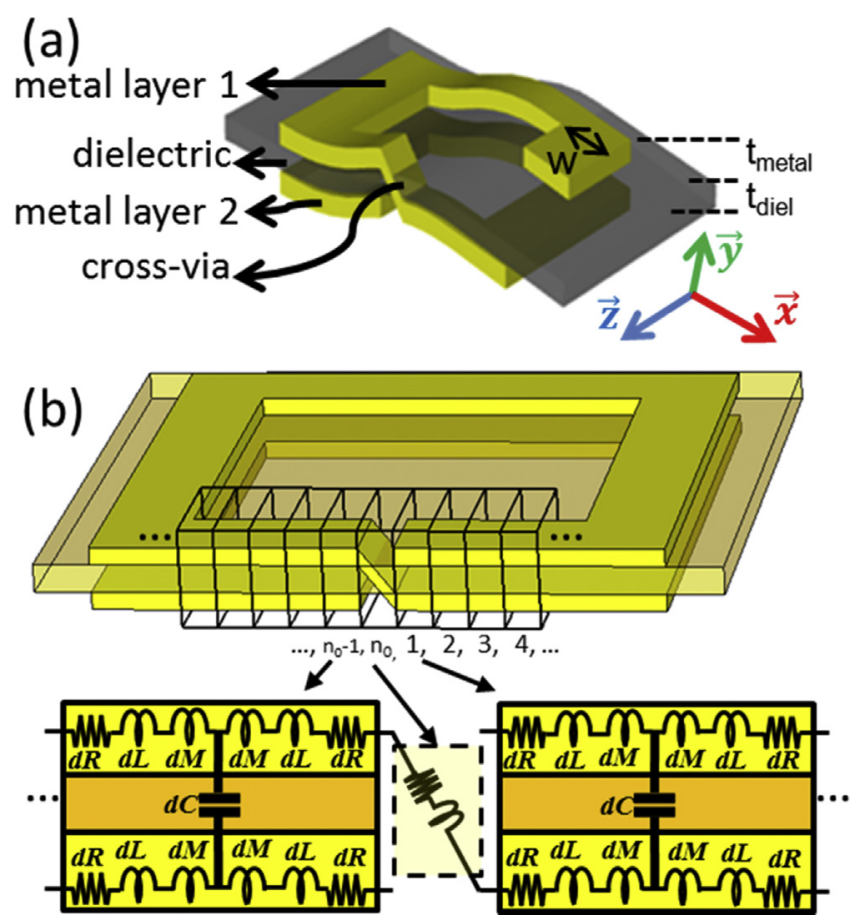

Fig. 1. Schematic representation of the proposed wireless metamaterial structure (not drawn to scale). (a) A metal-insulator-metal (MIM) device with cross-via metallization to increase $L_{\text {eff }}$ in a given footprint area (with semi-turn overlay). (b) The operation of the structure can be modeled by using a cascaded equivalent circuit approach. Each unit cell is composed of differential inductance $(d L)$, capacitance $(d C)$ and resistance $(d R)$ with a mutual coupling of $d M$ for partial overlay regions.

studied structures, this metamaterial architecture is composed of two inductive layers connected in series (via cross-via metallization) with strong capacitive coupling, resulting in distributed thin-film capacitance behavior that cannot be analyzed using conventional methods $[40,41]$.

Before we model the proposed architecture, we calculated the inductance of a single turn rectangular resonator, $L_{0}$, by using Eq. (3)

$L_{0}=0.635 \mu_{0} \mu_{r} n^{2} D_{a v}\left[\ln \left(\frac{2.07}{\rho}\right)+0.18 \rho+0.13 \rho^{2}\right]$

and the parallel plate capacitance, $C_{0}$, by using Eq. (4)

$C_{0}=\varepsilon_{0} \varepsilon_{r} \frac{A}{d}$

where $\mu_{0}$ is the permeability of the free space, $\mu_{\mathrm{r}}$ is the relative permeability of the material used for resonator fabrication (which has to be unity for MRI operation), $n$ is the number of turn (unity for a single layer structure), $D_{a v}$ is the average diameter $\left(D_{a v}=\frac{D_{0}+D_{i}}{2}\right)$, $\rho$ is the fill ratio $\left(\rho=\frac{D_{o}-D_{i}}{D_{0}+D_{i}}\right), D_{o}$ is the outer diameter (side length for rectangular resonators), $D_{i}$ is the inner diameter (side length$2 \times \mathrm{w}$ ) for the rectangular resonators), $w$ is the metallization width, $\varepsilon_{0}$ is the permittivity of the free space, $\varepsilon_{\mathrm{r}}$ is the relative permittivity of the dielectric used for electric field localization, $A$ is the parallel plate surface area and $d$ is the distance between the consecutive layers (e.g., dielectric thickness for the proposed structure).

Finally, the AC resistance of the single turn structure is calculated by using Eq. (5)

$R_{0}=\frac{l}{w \sigma \delta\left(1-e^{-t_{\text {metal }} / \delta}\right)}$

where $l$ is the meant path length of the metal trace (proportional to the number of turns, $n$ ), $t_{\text {metal }}$ is the thickness of the metallization, $\sigma$ is the conductivity of the metal used for fabrication and $\delta$ is the 
skin-depth of the metal for the given frequency that is formulated as given in Eq. (6).

$\delta=\sqrt{\frac{2}{2 \pi f \mu \sigma}}$

Stacking additional turns does not change the fill ratio, $\rho$. Thus, When we substitute Eqs. (3) and (5) into Eq. (2), we observe that the Q-factor is linearly proportional to the number of turns, $n$, for a pre-determined resonance frequency, $f_{0}$. This is not the case for spiral resonators because of decreasing mutual coupling among consecutive turns [33].

To model its behavior, we discretized the proposed structure to $n_{0}$ unit cells, as depicted in Fig. 1b; these unit cells are cascaded to construct the overall structure. Each unit cell is composed of differential inductance $\left(d L=L_{0} / 2 n_{0}\right)$, differential resistance $\left(d R=R_{0} / 2 n_{0}\right)$, differential thin-film capacitance $\left(d C=C_{0} / n_{0}\right)$ and differential mutual coupling $(d M, d M=k d L)$, where $k$ is the coupling coefficient and has a value of approximately 1 for strong positive coupling via cross-via connection [38]. For electrically small structures (e.g., electrical size $<\lambda_{0} / 300$ for this case), the corners of the proposed structure can also be modeled by using the above unit cell configuration. Similarly, the via metallization is also modeled as a series RL circuit (without $d C$ element) between consecutive layers.

By using Kirchhoff's current law, we constructed the admittance matrix as $V[Y]=I$, where $V$ and $I$ are the voltage and current vectors of the nodes respectively and $Y$ is the admittance matrix of the overall structure (details are reported in the supplementary file, section S1). The resulting impedance graphs are calculated by using a MATLAB ${ }^{\circledR}$ (The MathWorks, Inc. 01760 USA) to determine the resonance frequency (i.e., calculated from the peaks of the impedance graphs) and the Q-factor (calculated using the, full-width-half-maximum, FWHM, of the graphs) of the metamaterial for various $n_{0}$.

The effects of various geometric parameters, including $t_{\text {diel }}$ and overlay area, were analyzed by using a full-wave numerical solver, CST-Microwave Studio ${ }^{\text {TM }}$ (CST, 64289 Darmstadt, Germany). The simulation domain was composed of a cubic vacuum environment with a side length of $16 \mathrm{~mm}$ and the boundary conditions are set as perfectly matched layers (PMLs) in all directions. A square coil having a side length of $8 \mathrm{~mm}$, a trace width of $1 \mathrm{~mm}$, and a metallization thickness of $35 \mu \mathrm{m}$ is positioned $0.2 \mathrm{~mm}$ away from the proposed structure to be used as the pick-up coil to measure its input impedance. The metallization material used was gold for both the antenna and the resonator. The dielectric material of the proposed structure was chosen as lossy polyimide from the library of CST Microwave Studio, for full-wave solutions. The frequency domain solver was used to acquire the scattering parameters of the wireless structure. This simulation environment is depicted in Fig. 2. Here, we followed the methods described by Ginefri et al. [39] and measured the input impedance using a network analyzer (Agilent E5061B) and a pick-up coil antenna (details are reported in the supplementary file, section S2).

\subsection{Microfabrication of metastructures}

We fabricated two different devices (rigid and flexible ones) with different microfabrication methods. The rigid devices aim to achieve the smallest electrical footprint area (without targeting a predefined operational frequency) for a wireless metamaterial structure, where and the flexible device is microfabricated onto a polyimide substrate and tuned to a predefined MRI frequency. The rigid samples, presented in Fig. 3(a), are microfabricated onto a $<111>$ silicon substrate. By using a hard-mask with complementary SRR patterns, we thermally evaporated two $10 \mu \mathrm{m}$ thick $A u$ SRR layers (here gold is chosen because it is biocompatible) with a

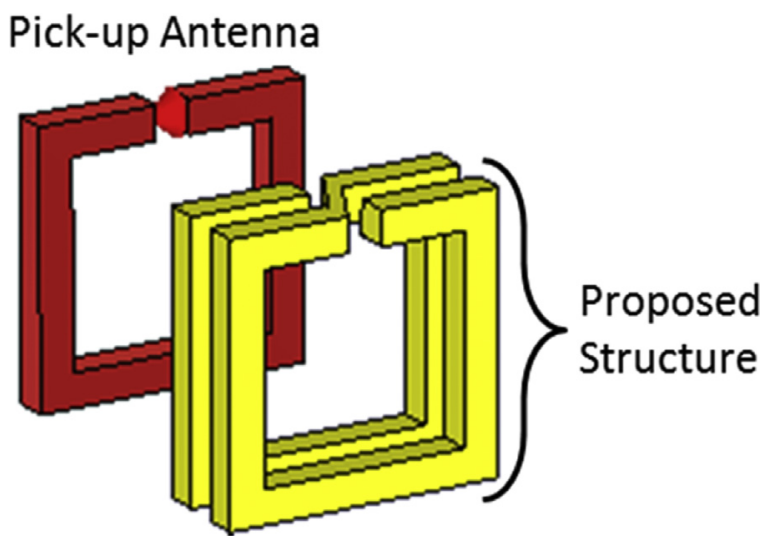

Fig. 2. Schematic representation of the measurement setup (not drawn to scale). The resonator is strongly coupled to the pick-up coil antenna to measure its characteristic properties.

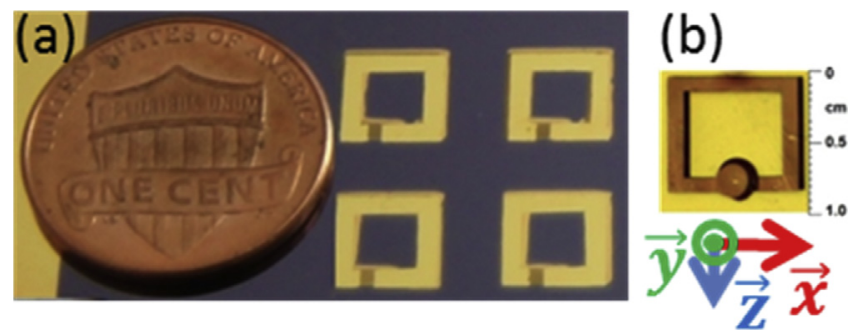

Fig. 3. Optical photographs of the microfabricated devices. (a) Rigid samples have the footprint of $6 \mathrm{~mm} \times 6 \mathrm{~mm}$ (for each feature). These samples are manufactured in array form onto a silicon wafer with a $1-\mu \mathrm{m}$ thick silicon nitride dielectric sandwiched between two $10-\mu \mathrm{m}$ thick gold layers. (b) The flexible structure is microfabricated onto a polyimide thin film with sub-cm dimensions $(8 \mathrm{~mm} \times 8 \mathrm{~mm}$ ).

$1 \mu \mathrm{m}$ thick plasma-enhanced-chemical-vapor-deposited (PECVD) silicon nitride $\left(\mathrm{Si}_{3} \mathrm{~N}_{4}\right)$ sandwiched between each of the gold layers.

The second sample, presented in Fig. 3(b), is microfabricated onto an $8 \mathrm{~mm} \times 8 \mathrm{~mm}$ footprint area by using a flexible polyimide film (Kapton ${ }^{\circledR}$ ) with an initial thickness of $7.5 \mu \mathrm{m}$. The proposed thin film is thinned to $2.7 \mu \mathrm{m}$ by using reactive ion etching (RIE), with the following recipe: $\mathrm{SF}_{6}: \mathrm{O}_{2}$ of $45: 15 \mathrm{sccm}$ at $150 \mathrm{~W} \mathrm{RF}$ power, with 25 mTorr pressure to increase the effective capacitance between consecutive layers. By using a hard-mask with complementary SRR patterns, we thermally evaporated two $10-\mu \mathrm{m}$ thick $A u$ SRR layers with asymmetric comb sizes, with one layer on each side of the polyimide film, and introduced via metallization through the substrate cross-connecting a pair of the opposite edges of each SRR on the other side. Subsequently, the samples were annealed at $250^{\circ} \mathrm{C}$ for $5 \mathrm{~min}$ for increased electrical conductivity. Unlike conventional optical lithography techniques used in rigid sample fabrication [40], this method prevents potential chemical hazards for biocompatibility and draws the simplest methodology for high yield fabrications.

\subsection{MRI setup}

To demonstrate the MRI operation of the proposed flexible metamaterial structure, we prepared a gel-phantom with dimensions $80 \times 80 \times 40 \mathrm{~mm}^{3}$ by using $1 \mathrm{~g} / \mathrm{L} \mathrm{NaCl}, 2.5 \mathrm{~g} / \mathrm{L} \mathrm{CuSO}{ }_{4}$ and $14 \mathrm{~g} / \mathrm{L}$ agarose-gel to mimic the tissue properties [41] with a corresponding relative permittivity of approximately 60 and a conductivity of $0.5 \mathrm{~S} / \mathrm{m}$. Two sets of evenly distributed 13 fiber pillars, each pillar with a diameter of $200 \mu \mathrm{m}$, were immersed into the pre-mentioned gel phantom and positioned along $\hat{z}$-direction with depths of 0.1 and $5.0 \mathrm{~mm}$ from the phantom surface (Fig. 4). Here we acquired the 2-D MR images perpendicular to the orientation of the pillar 


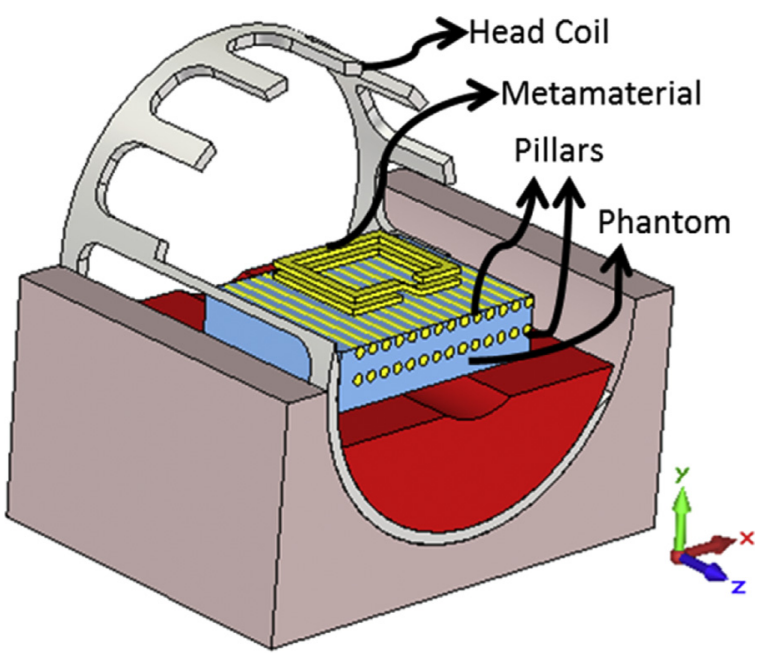

Fig. 4. Schematic representation of the MRI imaging setup (not drawn to scale). A phantom with two sets of pillars, where each set has 13 evenly distributed pillars with $200-\mu \mathrm{m}$ diameter and sets are separated by $5 \mathrm{~mm}$ apart from each other, is located inside a 12-channel head coil. The proposed metamaterial is positioned on top of the phantom to image the pillars in its vicinity.

array (i.e., transverse plane is the XY plane for $\hat{z}$-directed pillars) by using spoiled gradient echo (GRE) imaging sequence with an Echo Time (TE)/ Repetition Time (TR) of $9.25 \mathrm{~ms} / 100 \mathrm{~ms}$, a field of view (FOV) of $34 \times 34 \mathrm{~mm}$, an imaging matrix of $512 \times 512$, an imaging duration of $4 \mathrm{~min} 19 \mathrm{~s}$, a pixel bandwidth of $180 \mathrm{~Hz}$, a flip angle of $3^{\circ}$ and a slice thickness of $2 \mathrm{~mm}$. This imaging configuration corresponds to about $66 \mu \mathrm{m}$ spatial resolution at the imaging plane. All of the experiments were conducted by using standard twelve channel head coils of a $3 \mathrm{~T}$ Siemens Magnetom Tim-Trio scanner.

The working principle of the proposed metamaterial structure can be explained in two parts: 1) the inductive coupling of the transmit-field (body coils for this imaging configuration) and 2) the receive-field inductive coupling. This structure concentrates the flux in its vicinity that is transmitted during the excitation stage, which is called transmit-field coupling. In response to this over excitation, excited spins induces surface currents on this metamaterial and the metamaterial inductively transmits these signals to the receiver coils (head coils for this imaging configuration), which is called the receive-field coupling.

\section{Results and discussions}

\subsection{Resonance frequency and Q-factor calculations}

The Effective capacitance of the structure is strongly dependent on the dielectric thickness, $t_{\text {diel }}$, and the surface area of the thin-film loaded helical traces. This surface area, also called the overlay region, also affects the effective inductance, $L_{\text {eff }}$, because of increased or decreased the number of turns. Partial removal of one of the layers (e.g., the top layer is partially removed in Fig. 1) results in decreased $L_{\text {eff }}$ and $C_{\text {eff }}$, thereby increasing the increased resonance frequency. The effect of partial thin-film loading is numerically analyzed for different dielectric thicknesses by using CST Microwave Studio. These results are depicted in Fig. 5. The resonance frequency range of $65 \mathrm{MHz}$ to $5.5 \mathrm{GHz}$ is found to be achievable for the same footprint area with the given dielectric thicknesses (i.e., $8 \mathrm{~mm} \times 8 \mathrm{~mm}$ with 2.5 - $\mu \mathrm{m}$ thick polyimide).

Resonance frequency adjustment of self-resonant designs (i.e, designs that do not include lumped elements) to a predefined MRI frequency is not practical for most of the cases. Unlike the previously studied chiral metamaterial structures [42] or stacked-SRR type designs [43], here the proposed geometry allows us to eas-

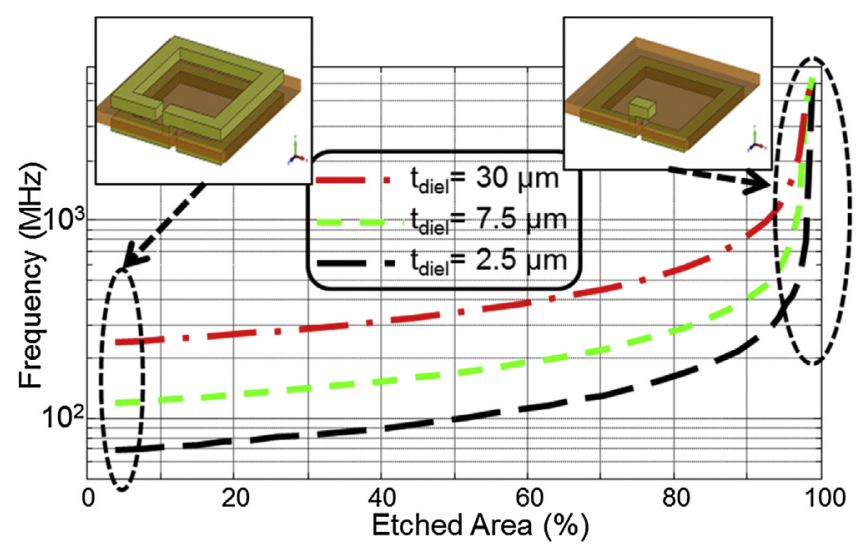

Fig. 5. Resonance frequency adjustment capability of the flexible design for $8 \mathrm{~mm} \times 8 \mathrm{~mm}$ footprint area (not drawn to scale). For fully loaded (double turn) helical ring geometry, it is possible to reach lower resonance frequencies. Partial removal of the metallization layer (upper layer for this configuration) results in increased resonance frequency via decreased effective inductance and capacitance. $100 \%$ percent etched area corresponds to a single layer SRR, where the resonance frequency increased to $5.5 \mathrm{GHz}$.

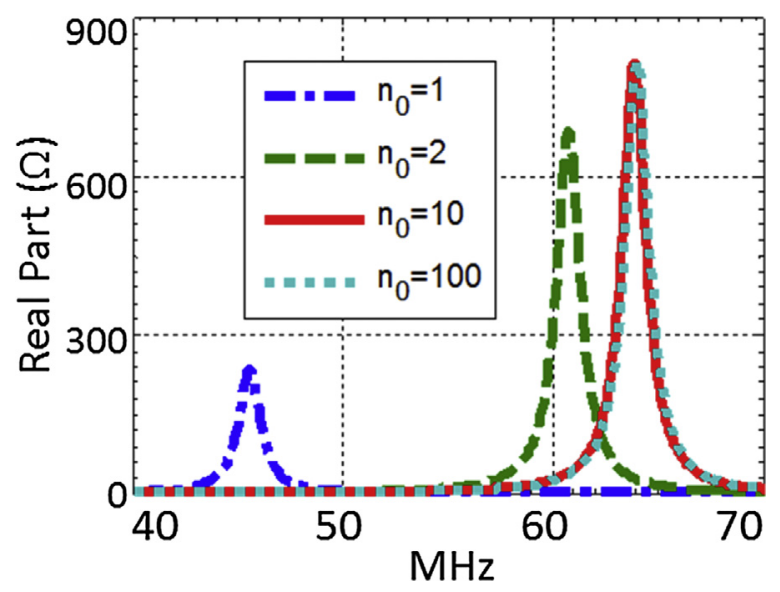

Fig. 6. Input impedance of the proposed metamaterial, with a footprint area of $8 \mathrm{~mm} \times 8 \mathrm{~mm}$ and dielectric thickness of $2.7 \mu \mathrm{m}$, for the given equivalent circuit model for various discretization number $n_{0}$. The results are convergent, and even using $n_{0}=10$ provides significantly accurate results.

ily increase the resonance frequency after its manufacturing, by using the metallization removal method for parallel plate coils [18]. As depicted in Fig. 5, removal of the metal overlay results in both decreased thin-film capacitance between consecutive metal layers and decreased effective-inductance due to lowered the number of turns. Hence, the resonance frequency of the proposed metamaterial structure can be conveniently increased to a predefined resonance frequency.

As proposed in Section 2.1, the equivalent circuit model for the given flexible metastructure with a footprint area of $8 \mathrm{~mm} \times 8 \mathrm{~mm}$ is analyzed to determine the resonance frequency and the Q-factor for various number of discretization. Fig. 6 depicts the impedance graphs for various discretization numbers, $n_{0}$. The acquired resonance frequencies and Q-factors calculated by using the FWHM of these graphs are also reported on Table 1.

Here, we observed that the increasing the discretization number of the proposed architecture converges to the $f_{0}$ of $64 \mathrm{MHz}$ and Q-factor of approximately 46, in reasonable agreement with the experimental results found as $65 \mathrm{MHz}$ and 42 for the resonance frequency and the $\mathrm{Q}$-factor respectively. By using conventional formulae (i.e. $\boldsymbol{f}_{0}=1 /\left(2 \pi \sqrt{\boldsymbol{L}_{\boldsymbol{e f f}} \boldsymbol{C}_{\boldsymbol{e f f}}}\right.$, with $\mathrm{L}_{0}=15.1 \mathrm{nH}, \mathrm{C}_{0}=303 \mathrm{pF}$, and $R_{0}=0.16 \Omega$ ), we calculated the $\mathrm{f}_{0}$ of $74.4 \mathrm{MHz}$ and a Q-factor 
Table 1

Performance metrics of the proposed metastructure, with a footprint area of $8 \mathrm{~mm} \times 8 \mathrm{~mm}$ and dielectric thickness of $2.7-\mu \mathrm{m}$, due to different discretization number $\left(\mathrm{n}_{0}\right)$.

\begin{tabular}{llll}
\hline $\mathrm{n}_{0}$ & $f_{0}(\mathrm{MHz})$ & Q-factor & Time $(\mathrm{s})$ \\
\hline 1 & 45 & 36 & 0,1 \\
2 & 60 & 44 & 0,1 \\
10 & 64 & 46 & 0,1 \\
100 & 64 & 46 & 3,1 \\
\hline
\end{tabular}
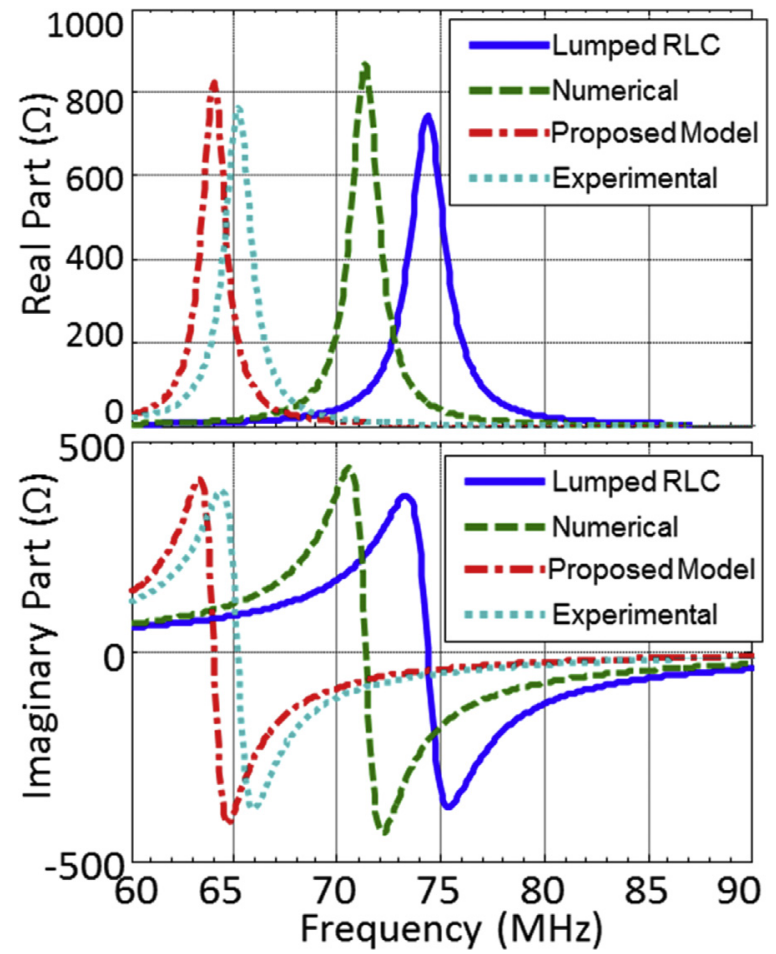

Fig. 7. Impedance graphs for the $8 \mathrm{~mm} \times 8 \mathrm{~mm}$ sample. Real (top) and imaginary (bottom) parts of the complex impedances show that the proposed equivalent circuit model estimates the resonance frequency and the Q-factor of the given structure better than the conventional lumped RLC model and has a performance similar to that of the full-wave numerical solutions.

of 36 for the proposed metastructure. Finally, $\mathrm{f}_{0}$ of $71 \mathrm{MHz}$ and Qfactor of 44 is obtained by using a numerical full wave solver. The impedance graphs of these results are shown in Fig. 7.

Similarly, for the structure with a footprint area of $6 \mathrm{~mm} \times 6 \mathrm{~mm}$, an inductance of $9.5 \mathrm{nH}$ and a capacitance of $1.6 \mathrm{nF}$ (for the given silicon-nitride dielectric of relative permittivity of 8.9 and loss tangent of 0.0006 ), and a resistance of $0.08 \Omega$ is used to calculate its operating frequency. The lumped RLC formulation resulted in $f_{0}$ of $41.2 \mathrm{MHz}$ and Q-factor of 20 , where proposed equivalent circuit model resulted in $\mathrm{f}_{0}$ of $35.4 \mathrm{MHz}$ and $\mathrm{Q}$-factor of 26 , which are in reasonable agreement with the experimental results of $33.4 \mathrm{MHz}$ and 13 for the resonance frequency and the Q-factor, respectively. Finally, $\mathrm{f}_{0}$ of $35.6 \mathrm{MHz}$ and Q-factor of 11 is obtained by using a numerical full wave solver. The impedance graphs of these results are visualized in Fig. 8.

We observed that the proposed equivalent circuit model estimates the resonance frequency of the metastructure, better than conventional calculation methods. Additionally, we see that the proposed thin-film loaded geometry neither behaves as a simple series resonator, nor like a parallel resonator: rather, it is a cascaded RLC circuit with proper feedback (i.e., feedback in electrical model corresponds to a physical connection called crossvia-metallization) to provide better resonance behavior. Hence, the proposed cascaded equivalent circuit model characterizes the
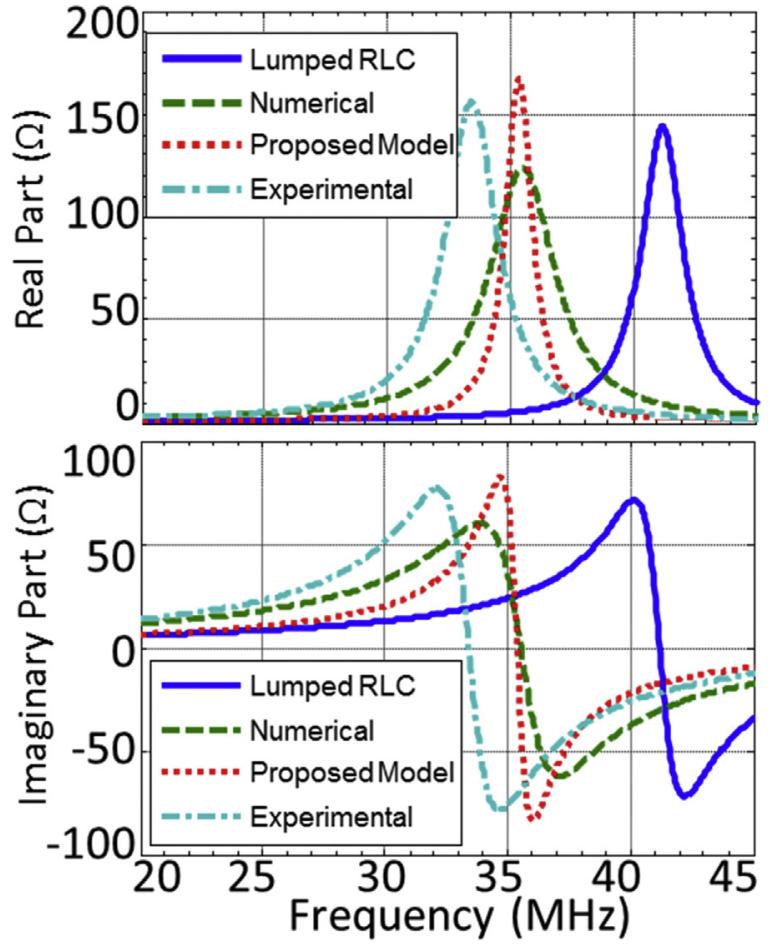

Fig. 8. Impedance graphs for the $6 \mathrm{~mm} \times 6 \mathrm{~mm}$ sample. Real (top) and imaginary (bottom) parts of the complex impedances show that the proposed equivalent circuit model estimates the resonance frequency and the $\mathrm{Q}$-factor of the proposed structure better than conventional lumped RLC model and has a performance similar to that of the numerical full-wave solver.

behavior of the structure more accurately compared to conventional lumped-element based methods. Because the proposed circuit model cannot include the effect of surrounding medium, which requires full-wave simulations, it cannot estimate the Qfactor correctly. Hence, calculation of Q-factor under lossy medium, such as loading, might not be reliable for the given circuit model, as is also the case for the conventional lumped RLC models.

In the impedance graphs presented in Fig. 8, we found the resonance frequency of the rigid metamaterial $(6 \mathrm{~mm} \times 6 \mathrm{~mm}$ resonator sandwiching a $1-\mu \mathrm{m}$ thick silicon-nitride) to be $33.4 \mathrm{MHz}$ with a corresponding free-space wavelength $\left(\lambda_{0}\right)$ of $8.98 \mathrm{~m}$. We calculated that the side length of the rigid resonator $(6 \mathrm{~mm})$ is shorter than $\lambda_{0} / 1500$, which is one of the smallest single-chip deepsubwavelength resonators reported thus far in the literature [44]. Although a theoretical work with a side length of $\lambda_{0} / 1733$ [45] and an experimental work with lumped capacitors having a side length of $\lambda_{0} / 2000$ [46]were reported, there is no experimental demonstration for a wireless self-resonant structure (i.e., without lumped element) electrically smaller than the structure presented here.

\subsection{Resonance frequency adjustment of the flexible sample to a pre-defined mri frequency}

To achieve the resonance frequency near a predefined MRI frequency (123 MHz for our $3 \mathrm{~T}$ MRI scanner) we used optical lithography to precisely etch the necessary amount of metal on the overlay. We observed that etching $17.5 \mathrm{~mm}^{2}$ on the overlay (corresponds to approximately $63 \%$ etched area of consecutive layers) resulted in a resonance frequency of $126 \mathrm{MHz}$, which was in agreement with the numerical results provided in Fig. 5. The measured Q-factor of the flexible metamaterial was also increased from 42 to approximately 82 , which was in agreement with $Q=2 \pi f_{0} L_{e f f} / R$ via the increased resonance frequency. It was previously reported that the Q-factor of conventional structures, (e.g., spirals, solenoids 
Table 2

Experimental characterization results for different samples.

\begin{tabular}{|c|c|c|c|c|}
\hline Type & Dielectric (thickness) & Etch Ratio (\%) & $\mathrm{f}_{0}(\mathrm{MHz})$ & Q-factor \\
\hline Rigid $\left(6 \times 6 \mathrm{~mm}^{2}\right)$ & $\mathrm{Si}_{3} \mathrm{~N}_{4}(1 \mu \mathrm{m})$ & 0 & 33.4 & 13 \\
\hline Flexible $\left(8 \times 8 \mathrm{~mm}^{2}\right)$ & Polyimide $(2.7 \mu \mathrm{m})$ & 0 & 65.0 & 42 \\
\hline Flexible $\left(8 \times 8 \mathrm{~mm}^{2}\right)$ & Polyimide $(2.7 \mu \mathrm{m})$ & 60 (tuned) & 126.0 & 82 \\
\hline Flexible-Loaded $\left(8 \times 8 \mathrm{~mm}^{2}\right)$ & Polyimide $(2.7 \mu \mathrm{m})$ & 60 (tuned) & 123.5 & 64 \\
\hline
\end{tabular}

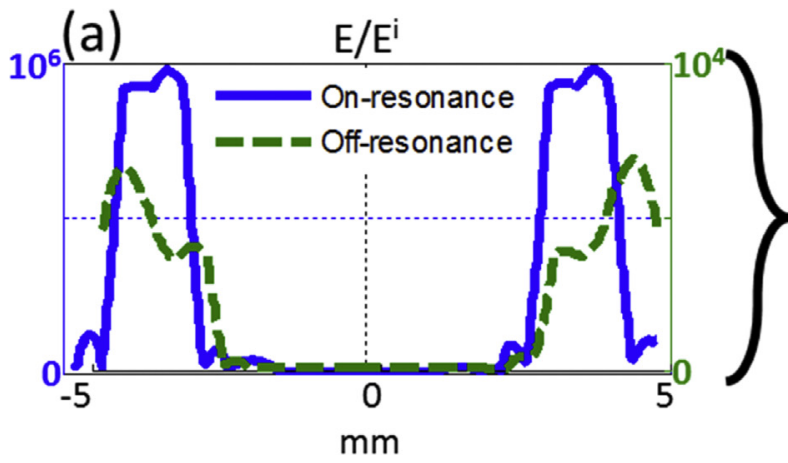

(b)

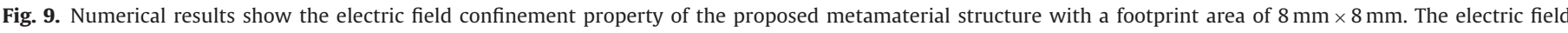

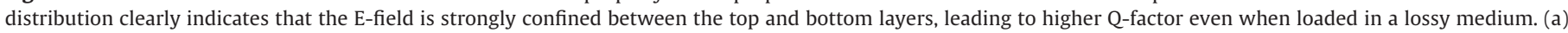

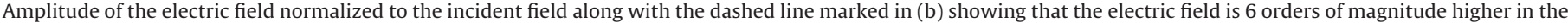
localized region on resonance with respect to the incident field.

and SRRs) do not increase linearly because of reduced inductance (and the reduced mutual inductance) for the consecutive turns [33]. However, the proposed geometry provides almost a linear Q-factor improvement, via the strong mutual coupling among consecutive layers. Thus, this structure is very special in terms of higher Q-factor and lowered resonance frequency for a smaller footprint area.

The tuned resonator was immersed into the abovementioned phantom, as described in Section 2.3, and its wireless impedance was measured by using the same pick-up coil and method [30]. The loaded Q-factor for this configuration was measured as 64 according to the FWHM of the complex impedance. The results are summarized in Table 2 for comparison.

\subsection{Electric field confinement for improved loading performance}

To permit use at extremely subwavelength frequencies, conventional resonators (such as SRRs, spirals etc.) require lumped capacitors to tune their resonance frequencies. The electric field distribution of these structure spills over from their plane because of these lumped capacitors, resulting in vulnerability to external effects such as loading with a lossy tissue (see details are reported in the supplementary file, Section S3). The use of the double-layer helical geometry provides an advantage of electric field localization in the lower-loss dielectric region (compared to living tissues) that allows for higher Q-factor. Fig. 9(a) presents the $|\vec{E}|$-field profile of the map normalized to incident field $\left(\vec{E}_{\dot{j}}\right)$ along with the dashed line marked in Fig. 9(b), where incident field is the electric field intensity recorded at the excitation port of the simulation domain. We observed that the electric field is 6 orders of magnitude higher in the localized region under resonance condition matched to the MRI frequency compared to the incident field and almost 3 orders of magnitude higher compared to the off-resonance case. Fig. 9(b) indicates the absolute value of the electric field distribution $(|\vec{E}|$ map) localized in the dielectric region between the top and bottom metallic layers. Clearly the electric field is strongly confined between the metallization lines; this strong confinement is essential to achieving high Q-factor even when loaded in a lossy medium including biological tissues.
Living tissues exhibit higher conductive losses, e.g., conductivity of $1 \mathrm{~S} / \mathrm{m}$, whereas the dielectrics have radically lower conductivities, e.g., conductivity of $10^{-16} \mathrm{~S} / \mathrm{m}$ for a Kapton ${ }^{\circledR}$. The proposed metamaterial device confines the electric field inside the dielectric material, instead of living tissues, thereby drastically decreasing the dissipated resistive power at the given frequency when the metastructure is placed in a lossy media. This property helps to maintain the Q-factor of the metamaterial device, even when the metastructure is loaded in vivo. Hence, the proposed deep-subwavelength metamaterial exhibits these properties for wireless operation that are critical to in vivo studies.

\subsection{MRI characterization}

The proposed structure was located on a coronal plane (XZ plane), where immersed pillars were aligned in the $\hat{z}$-direction, and the sample was located inside a standard head coil of the scanner, as shown in Fig. 10(a). Transverse images, with the imaging parameters given in Section 2.3, were acquired to count each and every pillar as shown in Fig. 10(b). Here, we observed that the pillar array can be visible only in the vicinity of the resonator, in agreement with the $\mathrm{B}_{1}{ }^{+}$map of the wireless metastructure as shown in Fig. 10 (c). The $\mathrm{B}_{1}{ }^{+}$results showed that the amplitude of the RF magnetic field can be amplified significantly in the vicinity of the resonator. From the MR image given in Fig. 10(b), we obtained the intensity plots along blue and red lines marked on it. From Fig. 10(b), we selected a region with a size of $100 \times 100$ pixels that does not contain any MRI source (i.e. noise region), and calculated the average noise level to be approximately 50 arbitrary units (a.u.), where the signal intensity in the neighboring region of the resonator appears to be approximately 1000 a.u. The intensity curve in blue, drops to approximately noise level for the corresponding pillars in the vicinity of the metamaterial resonator. We can clearly count the number of dips as 13 , which is the number of pillars in the array. The red curve (at $5 \mathrm{~mm}$ away) shows the intensity profile far away from the resonator (Fig. 10-d). Clearly, the pillars $5 \mathrm{~mm}$ away from the metamaterial are not visually separable from each other. 

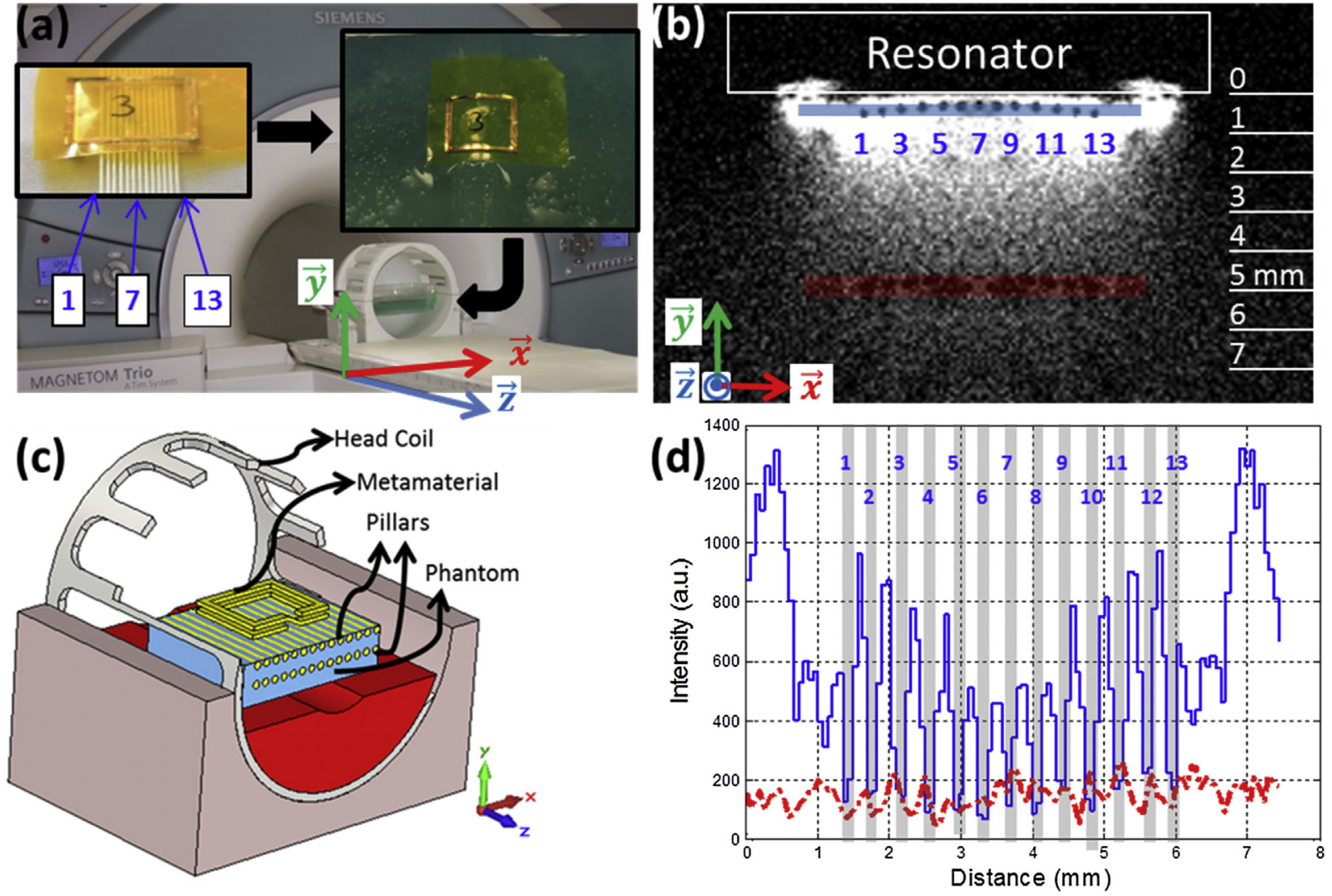

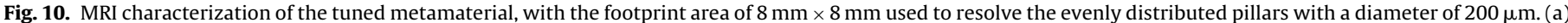

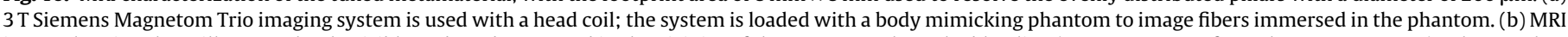

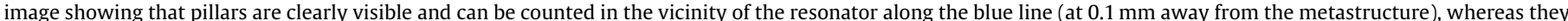

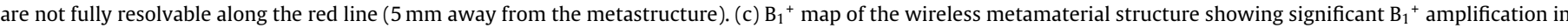

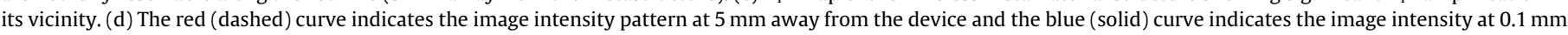

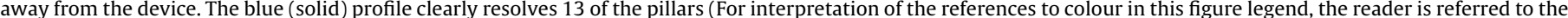
web version of this article).

Here we also see that the signal intensity is amplified approximately one order of magnitude $(1000 / 100)$ in the vicinity of the metastructure. For the pillars close to the resonator metallization, the contrast-to-noise-ratio (CNR) reaches to approximately 15.4 (e.g., $C N R=\frac{920-150}{50}$ ), where CNR drops to approximately 6.9 for the 6th and 7th pillars because of the increased SNR in the proximity of the meta-device, verified by its $\mathrm{B}_{1}{ }^{+}$map. For the pillars away from the resonator, the CNR drops to approximately unity, which is meaningless. To image small objects, e.g., pillars with $200 \mu \mathrm{m}$ diameter, the SNR should be kept high enough to eliminate noise effects. The proposed metamaterial structure manipulates the EM field strongly in its vicinity to increase the SNR, allowing for MRI of sub-mm pillars with an unprecedented resolution for $3 \mathrm{~T}$ head coils that is otherwise not possible using these imaging parameters.

\section{Conclusion}

In conclusion, we proposed, modeled and demonstrated a wireless compact metamaterial architecture with a side length of approximately $\lambda_{0} / 1500$ and frequency adjustment range of $65 \mathrm{MHz}$ to $5.5 \mathrm{GHz}$. This proposed metastructure possess a Q-factor of approximately 80 for $123 \mathrm{MHz}$. We demonstrated that this deepsubwavelength metamaterial can be used as an SNR-enhancing device for MRI. Because of its strong electric field confinement property in its dielectric region, the proposed structure exhibits higher Q-factors even in a lossy medium (i.e., living tissues). Therefore, this wireless metastructure is an excellent candidate for use in var- ious applications including in vivo MR imaging platforms and smart implants.

\section{Acknowledgements}

HVD gratefully acknowledges support from TÜBA. This work is partially supported by Turkish National Scientific and Technological Research Institute TÜBITAK-BIDEB. Authors of this work gratefully acknowledge the support of UNAM-National Nanotechnology Research Center and Institute of Materials Science and Nanotechnology.

\section{References}

[1] V.G. Veselago, The electrodynamics of substances with simultaneously negative values of $\varepsilon$ and $\mu$, Sov. Usp. 10 (1968) 509-514.

[2] W. Withayachumnankul, K. aruwongrungsee, A. Tuantranont, C. Fumeaux, D Abbott, Metamaterial-based microfluidic sensor for dielectric characterization, Sens. Actuators: Phys. 189 (2013) 233-237.

[3] A. Daliri, A. Galehdar, S. John, C.H. Wang, W.S.T. Rowe, K. Ghorbani, Wireless strain measurement using circular microstrip patch antennas, Sens. Actuators: Phys, 184 (2012) 86-92.

[4] R. Melik, E. Unal, N.K. Perkgoz, C. Puttlitz, H.V. Demir, Metamaterial-based wireless strain sensors, Appl. Phys. Lett. 95 (2009) 011106.

[5] A.K. Horestani, J. Naqui, Z. Shaterian, D. Abbott, C. Fumeaux, F. Martin, Two-dimensional alignment and displacement sensor based on movable broadside-coupled split ring resonators, Sens. Actuators: Phys. 210 (2014) 18-24.

[6] R. Melik, E. Unal, N.K. Perkgoz, C. Puttlitz, H.V. Demir, Flexible metamaterials for wireless strain sensing, Appl. Phys. Lett. 95 (2009) 181106.

[7] A. Alipour, E. Unal, S. Gokyar, H.V. Demir, Development of a distance-independent wireless passive RF resonator sensor and a new 
telemetric measurement technique for wireless strain monitoring, Sens Actuators: Phys. 255 (2017) 87-93.

[8] M. Lapine, D. Powell, M. Gorkunov, I. Shadrivov, R. Marqués, Y. Kivshar Structural tunability in metamaterials, APL 95 (2009) 084105, http://dx.doi. org/10.1063/1.3211920.

[9] F. Bilotti, L. Nucci, L. Vegni, An SRR based microwave absorber, Microw. Opt. Technol. Lett. 48 (11) (2006) 2171-2175.

[10] J.B. Pendry, Negative refraction makes a perfect Lens, Phys. Rev. Lett. 85 (2000) 3966.

[11] N. Fang, H. Lee, C. Sun, X. Zhang, Sub-Diffraction-limited optical imaging with a silver superlens, Science 308 (2005) 534.

[12] M.J. Freire, R. Marques, Near-field imaging in the megahertz range by strongly coupled magnetoinductive surfaces: experiment and ab initio analysis, J. Appl. Phys. 100 (2006) 063105.

[13] M.C.K. Wiltshire, J.B. Pendry, I.R. Young, D.J. Larkman, D.J. Gilderdale, J.V. Hajnal, Microstructured magnetic materials for RF flux guides in magnetic resonance imaging, Science 291 (2001), 849 - 51.

[14] M.J. Freire, R. Marques, L. Jelinek, Experimental demonstration of a $\mathrm{u}=-1$ metamaterial lens for magnetic resonance imaging, Appl. Phys. Lett. 93 (2008) 231108.

[15] E. Moser, M. Meyerspeer, F.Ph.S. Fischmeister, G. Grabner, H. Bauer, S. Trattnig, Windows on the human body-in vivo high-field magnetic resonance research and applications in medicine and psychology, Sensors 10 (2010) 5724-5757.

[16] M. Blaimer, F. Breuer, M. Mueller, R.M. Heidemann, M.A. Griswold, P.M. Jakob, SMASH, SENSE, PILS, GRAPPA, how to choose the optimal method, Top Mag. Reson. Imaging 15 (August) (2004) 223-236, Nu. 4.

[17] M.A. Griswold, P.M. Jakob, R.M. Heidemann, M. Nittka, V. Jellus, J. Wang, B. Kiefer, A. Haase, Generalized autocalibrating partially parallel acquisitions (GRAPPA), Magn. Reson. Med. 47 (2010) 1202-1210.

[18] P. Gonord, S. Kan, A. Leroy-Willig, Parallel plate Split-conductor surface coil: Analysis and design, Magn. Reson. Med. 6 (1998) 353-358.

[19] P. Gonord, S. Kan, A. Leroy-Willig, C. Wary, Multigap parallel-plate bracelet resonator frequency determination and applications, Rev. Sci. Instrum. 65 (1994) 3363, http://dx.doi.org/10.1063/1.1144573.

[20] T. Dohi, K. Matsumoto, I. Shimoyama, Flexible micro resonator for the magnetic resonance catheter, in: The 13th Int. Conf. on Solid-State Sensors, Actuators and Microsystems, Seoul, Korea, June 5-9, 2005.

[21] T. Matsunaga, Y. Matsuoka, S. Ichimura, Q. Wei, K. Kuroda, Z. Kato, M. Esashi, Y. Haga, Multilayered receive coil produced using a non-planar photofabrication process for an intraluminal magnetic resonance imaging, Sens. Actuators: Phys. (2018), http://dx.doi.org/10.1016/j.sna.2017.04.021.

[22] J. Gawlitza, M. Reiss-Zimmermann, G. Thörmer, A. Schaudinn, N. Linder, N. Garnov, L.C. Horn, D.H. Minh, R. Ganzer, J.U. Stolzenburg, T. Kahn, M. Moche, $\mathrm{H}$. Bussea, Impact of the use of an endorectal coil for $3 \mathrm{~T}$ prostate MRI on image quality and cancer detection rate, Nat., Sci. Rep. 7 (2017) 40640, http:// dx.doi.org/10.1038/srep40640.

[23] C. Baltes, N. Radzwill, S. Bosshard, D. Marek, M. Rudin, Micro MRI of the mouse brain using a novel $400 \mathrm{MHz}$ cryogenic quadrature RF probe, NMR Biomed. 22 (2009) 834-842, http://dx.doi.org/10.1002/nbm.1396.

[24] D. Sakellariou, G. Le Goff, J.F. Jacquinot, High-resolution, high-sensitivity NMR of nanolitre anisotropic samples by coil spinning, Nature 447 (7 June) (2007) 694-697.

[25] I. Lin, H.C. Yang, J.H. Chen, Diffusion tensor imaging using a high-temperature superconducting resonator in a $3 \mathrm{~T}$ magnetic resonance imaging for a spontaneous rat brain tumor, Appl. Phys. Lett. 102 (2013) 063701.

[26] E.M. Kardoulaki, R.R.A. Syms, I.R. Young, M. Rea, W.M.W. Gedroyc, Thin-film micro-coil detectors: Application in MR-thermometry, Sens. Actuators: Phys. 226 (2015) 48-58.

[27] R.R.R.A. Syms, I.R. Young, M.M. Ahmad, M. Rea, C.A. Wadsworth, S.D. Taylor-Robinson, Thin-film detector system for internal magnetic resonance imaging, Sens. Actuators: Phys. 163 (2010) 15-24.

[28] E. Laistler, M. Poirier-Quinot, S.A. Lambert, R.M. Dubuisson, O.M. Girard, E. Moser, L. Darrasse, J.C. Ginefri, In vivo MR imaging of the human skin at subnanoliter Resolution using a superconductor surface coil at 1.5 tesla, J. Magn. Reson. Imaging 41 (February (2)) (2015) 496-504, http://dx.doi.org/10. 1002/jmri.24549.

[29] P.A. Bottomley, Turning up the heat on MRI, J. Am. Coll. Radiol. 5 (7) (July 2008) 853-855

[30] J.C. Ginefri, A. Rubin, M. Tatoulian, M. Woytasik, F. Boumezbeur, B. Djemai, M. Poirier-Quinot, F. Lethimonnier, L. Darrasse, E. Dufour-Gergam, Implanted, inductively-coupled, rf coils fabricated on flexible polymeric material: Application to in vivo rat brain MRI at 7T, J. Magn. Reson. 224 (2012) 61-70.

[31] J.A. Lehmann-Horn, J.F. Jacquinot, J.C. Ginefri, C. Bonhomme, D. Sakellariou, Monolithic MACS micro resonators, J. Magn. Reson. 271 (2016) 46-51.

[32] R. Kriegl, J.C. Ginefri, M. Poirier-Quinot, L. Darrasse, S. Goluch, A. Kuehne, E. Moser, E. Laisler, Novel inductive decoupling technique for flexible transceiver arrays of monolithic transmission line resonators, Magn. Reson. Med. 73 (2015) 1669-1681.

[33] F. Bilotti, A. Toscano, L. Vegni, Design of spiral and multiple Split-ring resonators for the realization of miniaturized metamaterial samples, IEEE Trans. Antennas Propag. 55 (August (8)) (2007), http://dx.doi.org/10.1109/ TAP.2007.901950.

[34] D.R. Smith, J.B. Pendry, M.C.K. Wiltshire, Metamaterials and Negative Refractive Index, Science 305 (2004) 788.
[35] Q.Y. Tang, Y.M. Pan, Y.C. Chan, K.W. Leung, Frequency-tunable soft composite antennas for wireless strain sensing, Sens. Actuators: Phys. 179 (2012) $137-145$.

[36] J.D. Baena, J. Bonache, F. Martin, R. Marques Sillero, F. Falcone, T. Lopetegi, M.A.G. Laso, J. Garcia-Garcia, I. Gil, M.F. Portillo, M. Sorolla, Equivalent-circuit models for split-ring resonators and complementary split-ring resonators coupled to planar transmission lines, IEEE Trans.Microw. Theory Technol. MTT-53 (2005) 1451-1461.

[37] S.S. Mohan, M. Hershenson, Simple accurate expression for planar spiral inductances, IEEE J. Solid State Circuits 34-10 (October) (1999) 1419-1424.

[38] Inder Bahl, Lumped Elements for RF and Microwave Circuits", Artech House 2003.

[39] J.C. Ginefri, E. Durand, L. Darrasse, Quick measurement of nuclear magnetic resonance coil sensitivity with a single-loop probe, Rev. Sci. Instrum. 70 (1999) 4730

[40] A.L. Coutrot, E. Dufour-Gergam, E. Martincic, J.P. Gilles, J.P. Grandchamp, J.M. Quemper, A. Bosseboeuf, F. Alves, B. Ahamada, Electromagnetic micro-device realized by electrochemical way, Sens. Actuators A 91 (2001) 80-84.

[41] V. Acikel, O. Ulutan, A.C. Ozen, B. Akin, Y. Eryaman, E. Atalar, A novel MRI based electrical properties measurement technique, Proc. Intl. Soc. Mag. Reson. Med. (2013) 21.

[42] R. Marques, L. Jelinek, F. Mesa, Negative refraction from balanced quasi-planar inclusions, Microw. Opt. Technol. Lett. 49 (10) (2007).

[43] E. Ekmekci, K. Topalli, T. Akin, G. Turhan-Sayan, A tunable multi-band metamaterial design using micro-split SRR structures, Opt. Express 17 (18) (2009).

[44] Fabrice Lemoult, Nadege Kaina, Mathias Fink, Geoffroy Lerosey, Wave propagation control at the deep subwavelength scale in metamaterials, Nat. Phys. 9 (2013).

[45] X. Zhang, E. Usi, S.K. Khan, M. Sadatgol, D.O. Guney, Extremely sub-wavelength negative index metamaterial, Prog. Electromagnet. Res. 152 (2015) 95-104.

[46] C.P. Scarborough, Z.H. Jiang, D.H. Werner, C. Rivero-Baleine, C. Drake, Experimental demonstration of an isotropic metamaterial superlens with a negative unity permeability at 8.5 MHz, Appl. Phys. Lett. 101 (2012) 014101.

\section{Biographies}

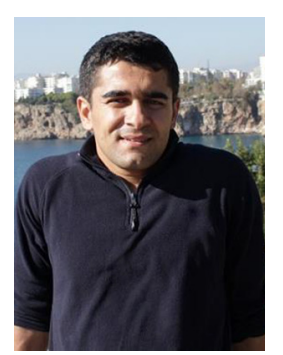

Sayim Gokyar completed his Ph.D. at Bilkent University, Department of Electrical and Electronics Engineering, Ankara. His research interests include wireless sensors and designing implantable electronic devices for wireless imaging applications as well as for sensing.

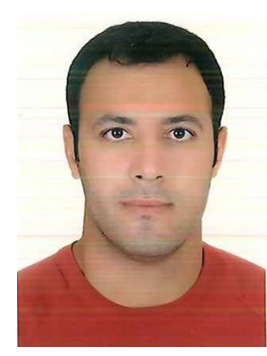

Akbar Alipour completed his Ph.D. at Bilkent University, Department of Electrical and Electronics Engineering Ankara. His research interests lie in the area of thin-film microwave structures which are used for magnetic resonance imaging (MRI) marking and wireless sensing. He has been involved in the development of devices which are mainly used in interventional MRI and medical implants.

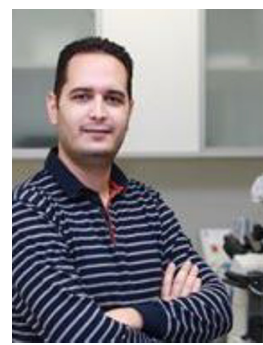

Emre Unal received his B.S. degree in electrical and electronics engineering from Hacettepe University, Ankara, Turkey, in 2005. He is a full-time Research Engineer under the supervision of Prof. H. V. Demir with the Institute of Materials Science and Nanotechnology, Bilkent University, Ankara, where he is working on the development of microwave and optoelectronic devices. 


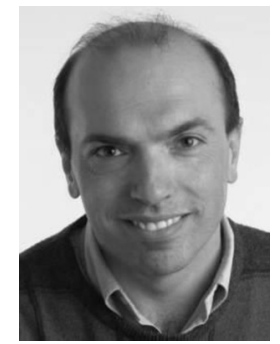

Ergin Atalar received his B.S. degree from Bogazici University in 1985, M. S. degree from Middle East Technical University in 1987, and Ph.D. degree from Bilkent University in 1991, all in Electrical Engineering. He joined the Johns Hopkins University, where he became a Professor of Radiology, Biomedical Engineering and Electrical and Computer Engineering and Director of Center for Image Guided Interventions. Currently, Dr. Atalar is a Professor of the Department of Electrical and Electronics Engineering and the Director of National Magnetic Resonance Research Center at Bilkent University. The main research interests of Dr. Atalar are Magnetic Resonance Imaging and TUBITAK science award.

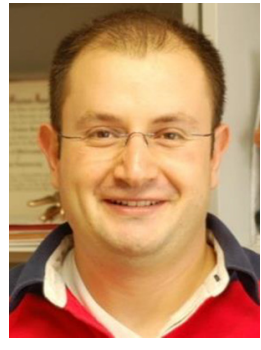

Hilmi Volkan Demir (M'04-SM'11) received the B.S degree in electrical and electronics engineering from Bilkent University, Ankara, Turkey, in 1998 and the M.Sc. and Ph.D. degrees in electrical engineering from Stanford University, Stanford, CA, in 2000 and 2004, respectively. In September 2004, he joined Bilkent University, where he is currently a professor with joint appointments at the Department of Electrical and Electronics Engineering and the Department of Physics and is also with UNAM- the Institute of Materials Science and Nanotechnology. Concurrently, he is a fellow of National Research Foundation in Singapore and a professor of Nanyang Technological University. His research interests include the development of innovative optoelectronic and RF devices. Prof. Demir is the recipient of the European Union Marie Curie Fellowship, the Turkish National Academy of Sciences Distinguished Young Scientist Award (TUBA-GEBIP), the European Science Foundation-European Young Investigator Award (ESF-EURYI), and Nanyang Award for Research Excellence. 